\title{
Transverse Pressure and Strangeness Dynamics in Relativistic Heavy Ion Reactions
}

\author{
M. Bleicher, E. Bratkovskaya, S. Vogel, X. Zhu \\ Institut für Theoretische Physik, J. W. Goethe Universität, 60054 Frankfurt am \\ Main, Germany \\ FIAS, Frankfurt Institute for Advanced Studies, Robert-Mayer-Str. 10, 60054 \\ Frankfurt am Main, Germany
}

\begin{abstract}
Transverse hadron spectra from proton-proton, proton-nucleus and nucleus-nucleus collisions from $2 \mathrm{AGeV}$ to $21.3 \mathrm{ATeV}$ are investigated within two independent transport approaches (HSD and UrQMD). For central $\mathrm{Au}+\mathrm{Au}(\mathrm{Pb}+\mathrm{Pb})$ collisions at energies above $E_{\mathrm{lab}} \sim 5 \mathrm{AGeV}$, the measured $K^{ \pm}$transverse mass spectra have a larger inverse slope parameter than expected from the default calculations. The additional pressure - as suggested by lattice QCD calculations at finite quark chemical potential $\mu_{q}$ and temperature $T$ - might be generated by strong interactions in the early pre-hadronic/partonic phase of central $\mathrm{Au}+\mathrm{Au}(\mathrm{Pb}+\mathrm{Pb})$ collisions. This is supported by a non-monotonic energy dependence of $v_{2} /\left\langle p_{T}\right\rangle$ in the present transport model.
\end{abstract}




\section{Introduction}

Recent lattice QCD (1QCD) calculations at vanishing quark chemical potential and finite temperature indicate critical energy densities for the formation of a quark-gluon plasma (QGP) of $\sim 0.6-1 \mathrm{GeV} / \mathrm{fm}^{3}$ [1]. Such energy densities might already be achieved at Alternating Gradient Synchrotron (AGS) energies of $\sim 10 \mathrm{AGeV}$ for central $\mathrm{Au}+\mathrm{Au}$ collisions [2, 3, 4]. According to lQCD calculations at finite quark chemical potential $\mu_{q}$ [5] a rapid increase of the thermodynamic pressure $P$ with temperature above the critical temperature $T_{c}$ for a cross over (or phase transition) to the QGP is expected.

Following the previous study [6] we speculate that partonic degrees of freedom might be responsible for this effect already at $\sim 5 A \cdot G e V$. Our arguments here are based on a comparison of the thermodynamic parameters $T$ and $\mu_{B}$ extracted from the transport models in the central overlap regime of $\mathrm{Au}+\mathrm{Au}$ collisions [7] with the experimental systematics on chemical freeze-out configurations [8] in the $T, \mu_{B}$ plane. The solid line in Fig. 1 characterises the universal chemical freeze-out line from Cleymans et al. [8] and the full dots with error bars denote the 'experimental' chemical freeze-out parameters - determined from the thermal model fits to the experimental particle ratios [8. The various smaller symbols (in vertical sequence) represent temperatures $T$ and chemical potentials $\mu_{B}$ extracted from UrQMD 1.3 transport calculations in central $\mathrm{Au}+\mathrm{Au}(\mathrm{Pb}+\mathrm{Pb})$ collisions at $\sqrt{s}=200 \mathrm{AGeV}, E_{\text {lab }}=160,40$ and $11 \mathrm{~A} \cdot \mathrm{GeV}$ [7] as a function of the reaction time in the center-of-mass (from top to bottom).

During the non-equilibrium phase (open symbols) the transport calculations show much higher temperatures (or energy densities) than the 'experimental' chemical freezeout configurations at all bombarding energies $(\geq 11 \mathrm{~A} \cdot \mathrm{GeV})$. These numbers are also higher than the tri-critical endpoints and phase boundary extracted from lattice QCD calculations by Karsch et al. [9] (large open circle) and Fodor and Katz [5] (star with horizontal error bar). Though the QCD lattice calculations differ substantially in the value of $\mu_{B}$ for the critical endpoint, the critical temperature $T_{c}$ is close to $160 \mathrm{MeV}$ in both calculations, while the energy density is of the order of $1 \mathrm{GeV} / \mathrm{fm}^{3}$ or even below. This diagram shows that at RHIC energies one encounters more likely a cross-over between the different phases when stepping down in temperature during the expansion phase of the hot fireball.

Indeed, a hardening of the measured transverse mass $\left(m_{t}\right)$ spectra in central $\mathrm{Au}+\mathrm{Au}$ collisions relative to pp interactions [10, 11] from AGS energies on is observed. This increase of the inverse slope parameter $T$ is commonly attributed to strong collective flow, which is absent in the respective pp or pA collisions. It has been proposed [12] to interpret the high and approximately constant $K^{ \pm}$slopes above $\sim 30 \mathrm{AGeV}$ - the 'step' - as an indication of the phase transition.

In this contribution we explore whether the pressure needed to generate a large collective flow to explain the hard slopes of the $K^{ \pm}$spectra with a 'plateau' at SPS energies is produced in the present transport models by interactions of hadrons or whether additional partonic contributions in the equation of state might be needed 


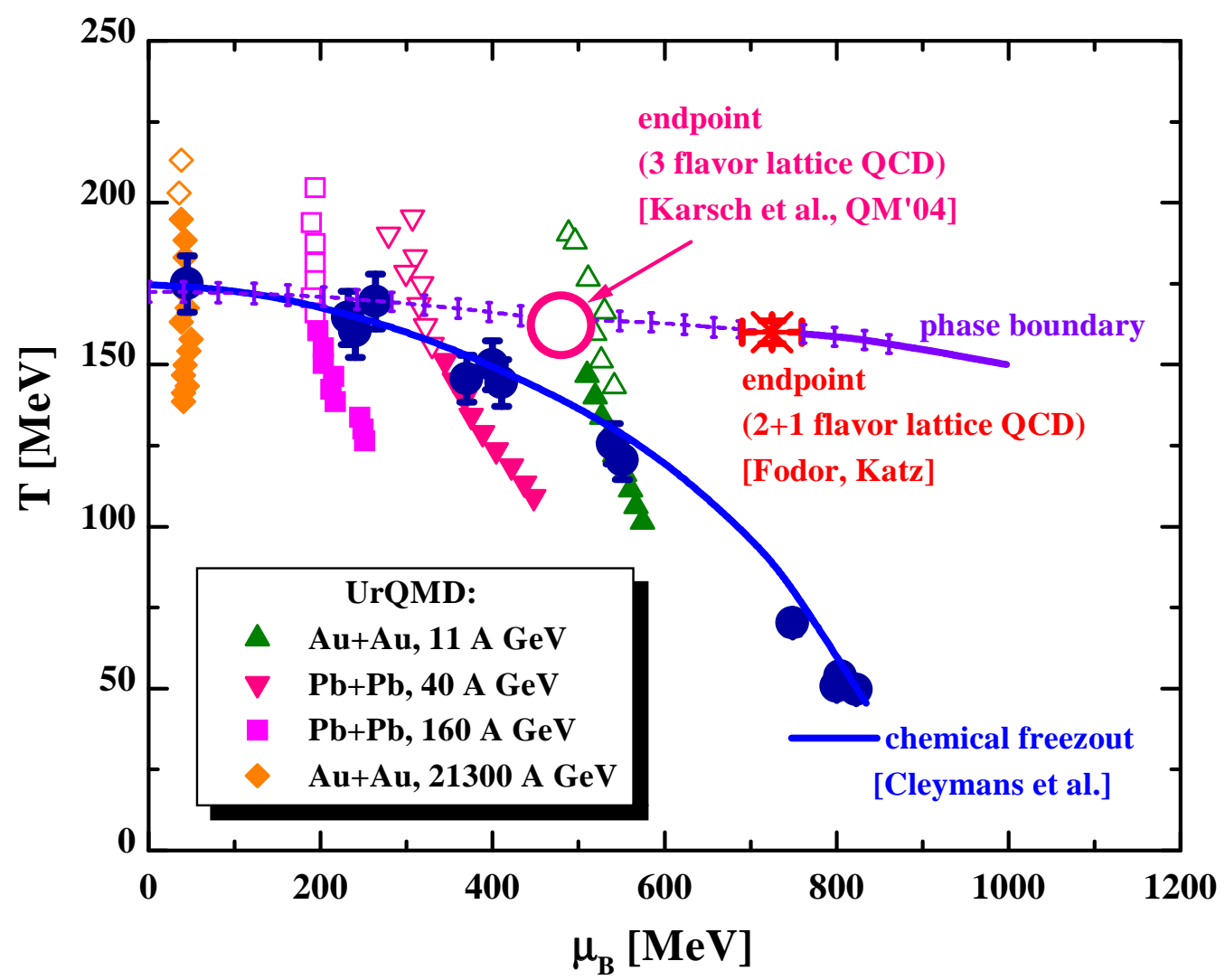

Figure 1. Schematic phase diagram in the $T-\mu_{B}$ plane. The solid line characterises the universal chemical freeze-out line from Cleymans et al. [8] and the full dots (with error bars) denote the 'experimental' chemical freeze-out parameters from Ref. 8]. The various symbols represent temperatures $T$ and chemical potentials $\mu_{B}$ extracted from UrQMD 1.3 transport calculations in central $\mathrm{Au}+\mathrm{Au}(\mathrm{Pb}+\mathrm{Pb})$ collisions at 21.3 $\mathrm{A} \cdot \mathrm{TeV}, 160,40$ and $11 \mathrm{~A} \cdot \mathrm{GeV}$ [7 (see text). The large open circle and the star indicate the tri-critical endpoints and phase boundary from lattice QCD calculations by Karsch et al. 9] and Fodor and Katz [5], respectively. The horizontal line with error bars is the phase boundary from [5].

to explain these effects (for further details the reader is referred to [13]). To understand whether a failure of the present models indeed hints a QGP onset, we explore two distinct effects that might result in a substantial increase of the transverse pressure: I) initial state Cronin enhancement and II) heavy resonance formation.

\section{The Models}

In our studies we use two independent relativistic transport models that employ hadronic and string degrees of freedom: UrQMD [14, 15] and HSD [16, 17. They take into account the formation and multiple rescattering of hadrons and dynamically describe the generation of pressure in the hadronic expansion phase. This involves also interactions of leading pre-hadrons that contain a valence quark (antiquark) from a primary 'hard' collision (cf. Refs. [18, 19]). Note that, in these models, only hadrons, valence quarks and valence diquarks and their interactions are treated explicitly. Gluonic 
degrees of freedom are not treated explicitly, but are implicitly present in strings. This simplified treatment is generally accepted to describe proton-proton and proton-nucleus interactions. Here we test whether this description is still valid for the more complicated nucleus-nucleus collisions, where large energy densities can be reached over extended volumes.

\section{Transverse Dynamics in Small and Large Systems}

Let us start by "benchmarking" the model calculations with pA data. Fig. 2 shows the results for the inverse slope parameters $T$ for various reactions - see figure caption for details. It can be seen that the models reproduce the transverse slope parameters of different particles produced in $\mathrm{pA}$ interactions with targets from Be to $\mathrm{Pb}$ reasonably well.

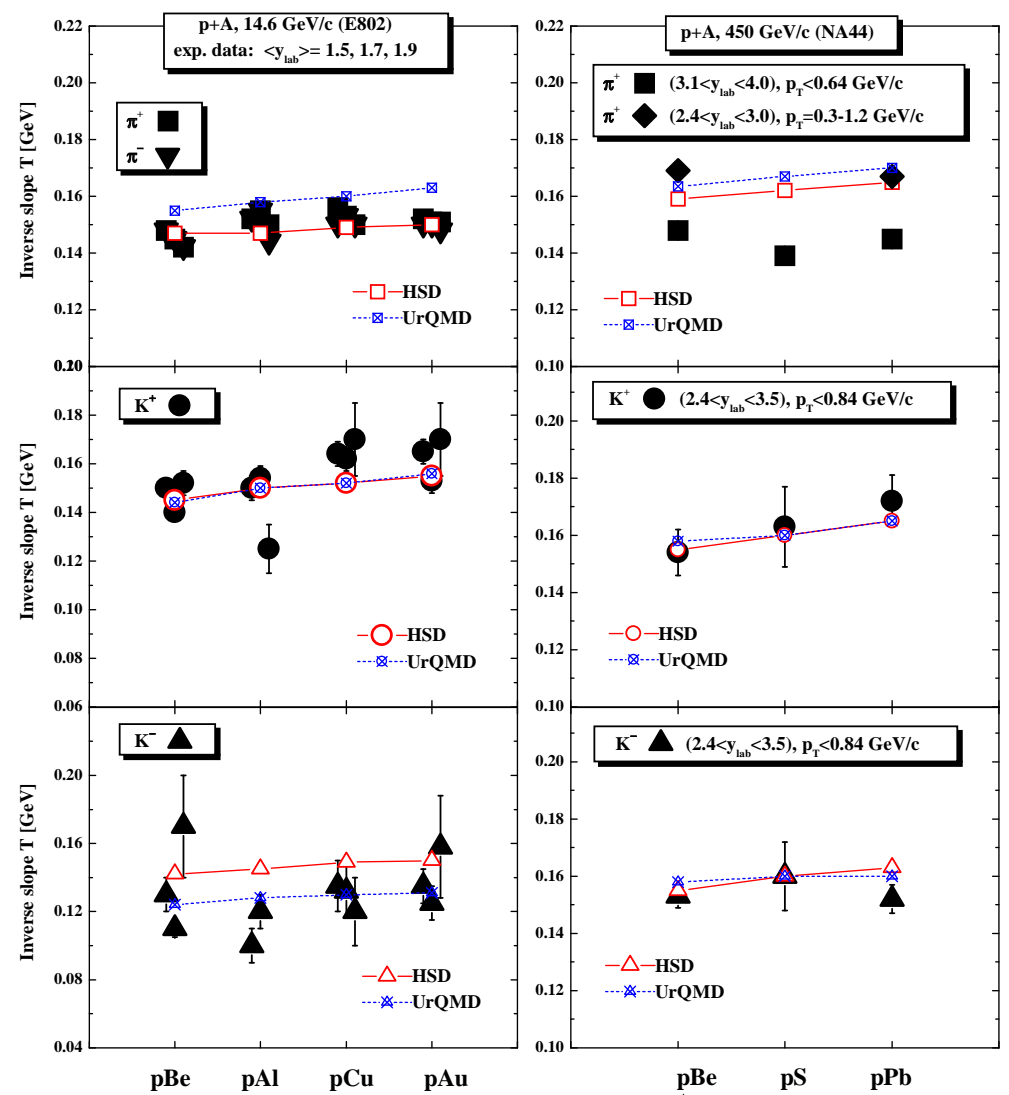

Figure 2. Inverse slope parameters $T$ for $\pi^{ \pm}, K^{+}$and $K^{-}$at midrapidity from pA reactions at $14.6 \mathrm{GeV} / c(\mathrm{~A}=\mathrm{Be}, \mathrm{Al}, \mathrm{Cu}, \mathrm{Au})$ - left part and at $450 \mathrm{GeV} / c(\mathrm{~A}=\mathrm{Be}$, $\mathrm{S}, \mathrm{Pb}$ ) - right part, from HSD (open symbols) and UrQMD 2.0 (closed symbols). The full symbols in the left part correspond to the midrapidity data $\left(\left\langle y_{l a b}\right\rangle=1.5,1.7,1.9\right)$ from the E802 Collaboration [27], in the right part to the NA44 data 28] at $2.4 \leq$ $y_{l a b} \leq 3.5, p_{T} \leq 0.84 \mathrm{GeV} / c$ for $K^{+}, K^{-}$and at $2.4 \leq y_{l a b} \leq 3.0, p_{T}=0.3 \div 1.2$ $\mathrm{GeV} / c$ (full diamonds) and $3.1 \leq y_{l a b} \leq 4.0, p_{T} \leq 0.64 \mathrm{GeV} / c$ (full squares) for $\pi^{+}$.

We continue with nucleus-nucleus collisions, where Fig. 3 summarises our results: the dependence of the inverse slope parameter $T$ on $\sqrt{s}$ is shown and compared to (partly 
preliminary) experimental data from [10, 20, 21, 22, 23, 24] for central $\mathrm{Au}+\mathrm{Au}(\mathrm{Pb}+\mathrm{Pb})$ collisions (1.h.s.) and [22, 25, 26] for pp collisions (r.h.s.). The upper and lower solid lines (with open circles) on the l.h.s. in Fig. 3] correspond to results from HSD calculations, where the upper and lower limits are due to fitting the slope $T$, an uncertainty in the repulsive $K^{ \pm}$-pion potential or the possible effect of string overlaps. The solid lines with stars correspond to HSD calculations with the Cronin effect. The dashed lines with open triangles represent slope parameters from UrQMD 1.3, the dot-dashed lines with open inverted triangles correspond to UrQMD 2.0 results, which are well within the limits obtained from the different HSD calculations without the Cronin enhancement. The dotted lines with crosses show the UrQMD 2.1 results that incorporate high mass resonance states up to $m_{R} \leq 3 \mathrm{GeV}$.

The slope parameters from pp collisions (r.h.s. in Fig. 3) are seen to increase smoothly with energy both in the experiment (full symbols) and in the HSD calculations (full lines with open circles). The UrQMD 1.3 results are shown as open triangles connected by a solid line and systematically lower than the slopes from HSD at all energies. When including jet production and fragmentation via PYTHIA in UrQMD 2.0 (dot-dashed lines with open inverted triangles) the results become similar to HSD above $\sqrt{s}=10 \mathrm{GeV}$ demonstrating the importance of jets in pp reactions at high energy.

Coming back to the slope parameters of $K^{ \pm}$mesons for central $\mathrm{Au}+\mathrm{Au} / \mathrm{Pb}+\mathrm{Pb}$ collisions (1.h.s. of Fig. 3) we find that the Cronin initial state enhancement indeed improves the description of the data at RHIC energies, however, does not give any sizeable enhancement at AGS energies. Here UrQMD 2.1 (dashed lines with crosses) with the high mass resonance states performs better: Including high mass resonances one obtains more reasonable results for $K^{+}$mesons, however, fails by 10 to $15 \%$ for pions as well as anti-Kaons. In this context it is interesting to note that the experimental results on $\mathrm{C}+\mathrm{C}$ and $\mathrm{Si}+\mathrm{Si}$ at $158 \mathrm{AGeV}$ show small slopes [25] and are therefore in agreement with the models [6].

What is the origin of the rapid increase of the slopes with energy for central $\mathrm{Au}+\mathrm{Au}$ collisions at AGS energies and the constant slope at low SPS energies (the 'step' in the Kaon temperature), which is missed in presently employed transport approaches?

\section{Elliptic Flow}

To disentangle the effects of high mass hadron states from a possible phase transition scenario we suggest to study the energy excitation function of the elliptic flow of pions (or negatively charged hadrons). The $\left\langle p_{T}\right\rangle$ and $v_{2}$ excitation functions are depicted in Fig. 4 (left). One clearly observes a monotonic rise in the mean transverse momentum of the pions with increasing energy. However, the elliptic flow behaves non-monotonic and shows a distinct maximum around 30-40 GeV beam energy. This phenomenon can be pronounced more clearly by the scaled elliptic flow $\left(v_{2} /\left\langle p_{T}\right\rangle\right)$ as shown in Fig. 4 (right). It was pointed out [29, 30] that at high energies the differential $v_{2}\left(p_{T}\right)$ of charged hadrons is approximately proportional to $p_{T}$, such that the averaged $v_{2} \propto\left\langle p_{T}\right\rangle$. In fact, when 

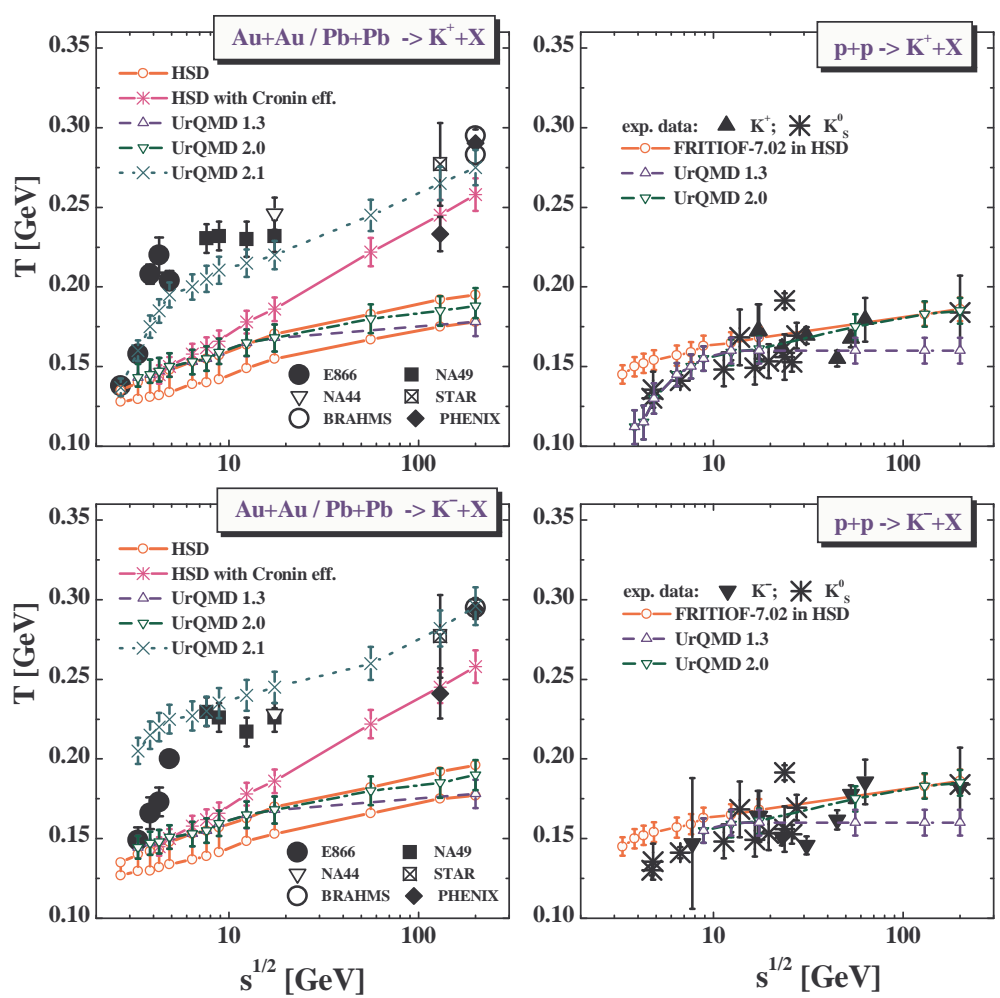

Figure 3. Comparison of the inverse slope parameters $T$ for $K^{+}$and $K^{-}$mesons from central $\mathrm{Au}+\mathrm{Au}(\mathrm{Pb}+\mathrm{Pb})$ collisions (l.h.s.) and pp reactions (r.h.s.) at midrapidity as a function of the invariant energy $\sqrt{s}$ from HSD (upper and lower solid lines with open circles), UrQMD 1.3 (dashed lines with open triangles), UrQMD 2.0 (dot-dashed lines with open inverted triangles), UrQMD 2.1 (dotted lines with crosses) with the data from Refs. [10, 20, 21, 22, 23, 24, for AA and 22, 25, 26] for pp collisions The upper and lower solid lines in the left diagrams result from different limits of the HSD calculations as discussed in the text while the solid lines with stars correspond to HSD calculations with the Cronin initial state enhancement.

divided by the average transverse momentum, the scaled elliptic flow in that energy regime becomes nearly constant [30, 31], even though the measured $v_{2}$ increases from SPS energy to RHIC energy.

To emphasise deviations from the natural scaling $v_{2} \propto\left\langle p_{T}\right\rangle$, we plot the excitation

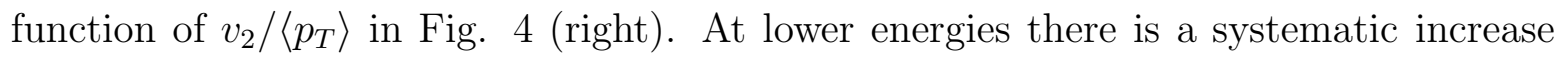
of $v_{2}$ relative to $\left\langle p_{T}\right\rangle$ in the model and in the data. However, above the SPS energy regime, one clearly observes that the data for the scaled elliptic flow is constant and independent of energy while the model yields first a sharp decrease of the $v_{2} /\left\langle p_{T}\right\rangle$ which then levels-off at roughly half the experimentally observed value.

The present non-equilibrium study however, suggest that the initial increase of the scaled elliptic flow up to SPS energies, might be due to viscosity effects (decreasing mean-free-path) in the hadronic gas. At higher energies, the predicted elliptic flow breaks down in the model calculation because of the increasing dominance of string dynamics. The measured data however, supports a hydrodynamical behaviour of the matter in the early stage with very small mean free paths. It should be noted that a 
similar minimum in the elliptic flow was first predicted by hydrodynamics. In contrast to the scenario discussed here, this decrease of $v_{2}$ was associated with the softening of the equation of state in the phase transition region [32, 33].

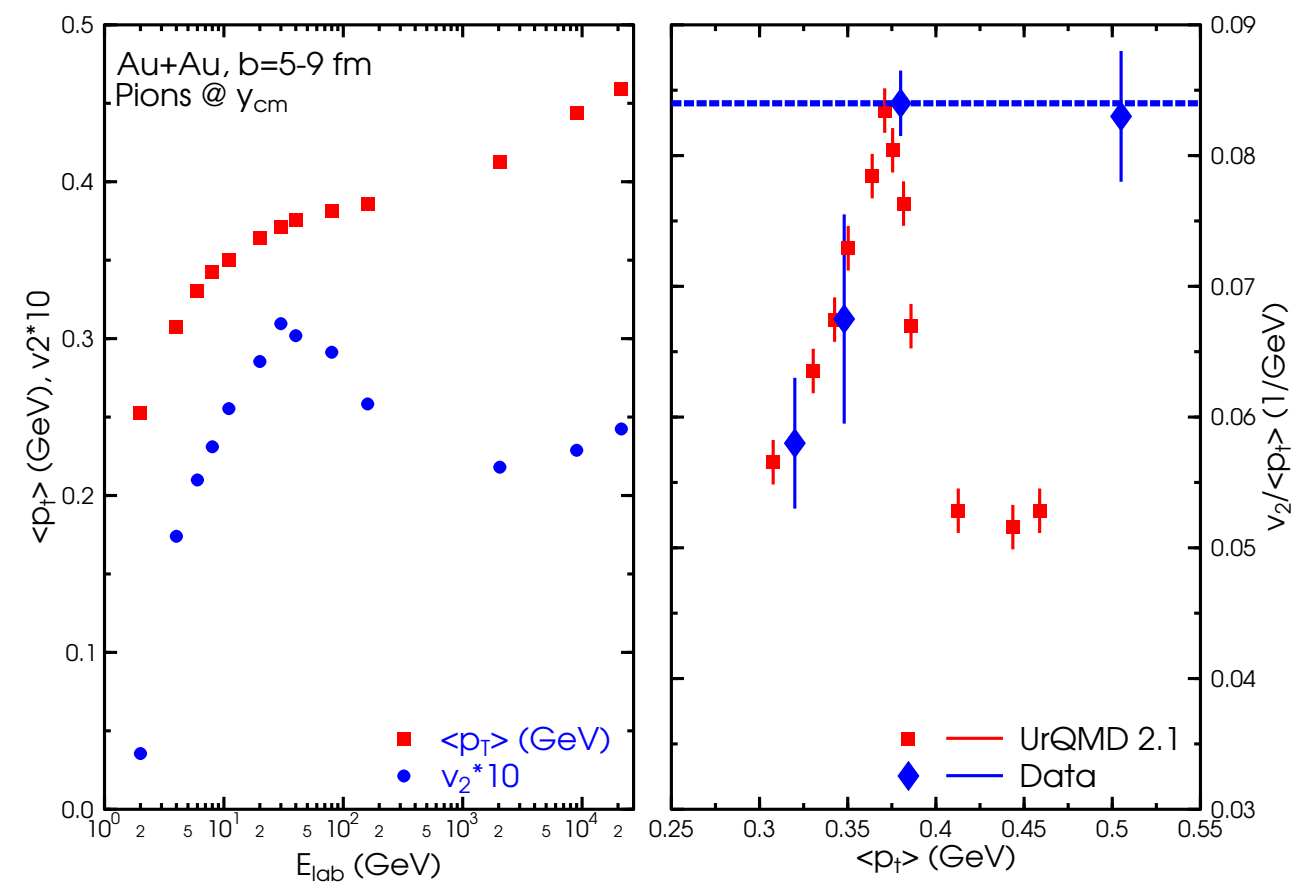

Figure 4. Left: Calculated excitation functions of mean transverse momentum and elliptic flow of pions at midrapidity. Right: Excitation function of $v_{2} /\left\langle p_{T}\right\rangle$ of Pions in mid-central collisions from top AGS to RHIC energy from UrQMD (squares). Negatively charged particle data (diamonds) for $v_{2}$ are taken from Ref. 34 and $\left\langle p_{T}\right\rangle$ from Refs. 35, 36, 37, 38.

Thus, from the lack of initial pressure we conclude that the system (at least at RHIC energies) seems to spend a considerable amount of time in the QGP phase with an equation of state harder than the employed hadron/string gas equation of state. This argument is well in line with the studies on elliptic flow at RHIC energies, which is underestimated by $\sim 30 \%$ at midrapidity in HSD [39] and by a factor of $\sim 2$ in UrQMD 1.3 [40]. It is our opinion that strong pre-hadronic/partonic interactions might cure this problem.

\section{Conclusion}

In conclusion, we have found that the inverse slope parameters $T$ for $K^{ \pm}$mesons from the HSD and UrQMD 1.3 transport models are practically independent of system size from pp up to central $\mathrm{Pb}+\mathrm{Pb}$ collisions and show only a slight increase with collision energy. The calculated transverse mass spectra are in reasonable agreement with the 
experimental results for pp reactions at all bombarding energies investigated as well as central collisions of light nuclei $(\mathrm{C}+\mathrm{C}$ and $\mathrm{Si}+\mathrm{Si}$ ) (cf. Ref. [6]). The rapid increase of the inverse slope parameters of Kaons for central collisions of heavy nuclei $(\mathrm{Au}+\mathrm{Au}$ or $\mathrm{Pb}+\mathrm{Pb}$ ) found experimentally in the AGS energy range, however, is not reproduced by both models in their default version (see Fig. 3).

We have discussed scenarios to improve the description of the experimental data. However, no fully convincing results could be obtained for all observables and bombarding energies simultaneously.

From comparison to lattice QCD calculations at finite temperature and baryon chemical potential $\mu_{B}$ from Refs. [5] and 9] as well as the experimental systematics in the chemical freeze-out parameters (cf. Fig. 1), we infer that the missing pressure above $30 \mathrm{GeV}$ beam energy might be generated in the early phase of the collision by non-perturbative partonic interactions. However, to fully clarify this issue will require a systematic quantitative comparison with hydrodynamic models from the lowest AGS energy to the highest RHIC energy.

\section{Acknowledgements}

This work was supported by GSI, DFG and BMBF. This work used computational resources provided by the Center for Scientific Computing at Frankfurt (CSC).

\section{References}

[1] F. Karsch et al., Nucl. Phys. B 502, 321 (2001).

[2] H. Stöcker and W. Greiner, Phys. Rep. 137, 277 (1986).

[3] W. Cassing et al., Nucl. Phys. A 674, 249 (2000).

[4] H. Weber, C. Ernst, M. Bleicher et al., Phys. Lett. B 442, 443 (1998).

[5] Z. Fodor and S. D. Katz, JHEP 0203, 014 (2002); Z. Fodor, S. D. Katz, and K. K. Szabo, Phys. Lett. B 568, 73 (2003).

[6] E. L. Bratkovskaya, S. Soff, H. Stöcker, M. van Leeuwen, and W. Cassing, Phys. Rev. Lett. 92, 032302 (2004).

[7] L. V. Bravina et al., Phys. Rev. C 60, 024904 (1999). Nucl. Phys. A 698, 383 (2002).

[8] J. Cleymans and K. Redlich, Phys. Rev. C 60, 054908 (1999).

[9] F. Karsch, talk given in Quark Matter 2004, Oakland, January 11-17, 2004.

[10] V. Friese et al., NA49 Collaboration, J. Phys. G 30, 119 (2004).

[11] M. I. Gorenstein, M. Gaździcki, and K. Bugaev, Phys. Lett. B 567, 175 (2003).

[12] M. Gazdzicki and M. I. Gorenstein, Acta Phys. Polon. B 30, 2705 (1999).

[13] E. L. Bratkovskaya et al., Phys. Rev. C 69, 054907 (2004) arXiv:nucl-th/0402026.

[14] S.A. Bass et al., Prog. Part. Nucl. Phys. 42, 255 (1998).

[15] M. Bleicher et al., J. Phys. G 25, 1859 (1999).

[16] J. Geiss, W. Cassing, and C. Greiner, Nucl. Phys. A 644, 107 (1998).

[17] W. Cassing and E. L. Bratkovskaya, Phys. Rep. 308, 65 (1999).

[18] H. Weber, E. L. Bratkovskaya, W. Cassing, and H. Stöcker, Phys. Rev. C 67, 014904 (2003).

[19] W. Cassing, K. Gallmeister, and C. Greiner, Nucl. Phys. A 735, 277 (2004)

[20] L. Ahle et al., E866 and E917 Collaboration, Phys. Lett. B 476, 1 (2000); ibid. 490, 53 (2000); Phys. Rev. C 58, 3523 (1998).

[21] I.G. Bearden et al., NA44 Collaboration, nucl-ex/0202019. 
[22] C. Adler et al., STAR Collaboration, nucl-ex/0206008 O. Barannikova et al., Nucl. Phys. A 715, 458 (2003); K. Filimonov et al., hep-ex/0306056.

[23] D. Ouerdane et al., BRAHMS Collaboration, Nucl. Phys. A 715, 478 (2003); J. H. Lee et al., J. Phys. G 30, S85 (2004).

[24] S. S. Adler et al., PHENIX Collaboration, nucl-ex/0307010 nucl-ex/0307022

[25] I. Kraus et al., NA49 Collaboration, J. Phys. G 30, 5583 (2004).

[26] M. Kliemant, B. Lungwitz, and M. Gaździcki, Phys. Rev. C 69, 044903 (2004)

[27] T. Abbott et al., E802 Collaboration, Phys. Rev. D 45, 3906 (1992).

[28] H. Boggild et al., NA44 Collaboration, Phys. Rev. C 59, 328 (1999).

[29] P. Huovinen, P. F. Kolb and U. W. Heinz, Nucl. Phys. A 698 (2002) 475 arXiv:nucl-th/0104020.

[30] R. Snellings, Talk presented at the 'Transverse Dynamics at RHIC' workshop, BNL, Upton, New York, 2003

[31] K. Paech, H. Stocker and A. Dumitru, Phys. Rev. C 68 (2003) 044907 arXiv:nucl-th/0302013.

[32] P. F. Kolb, J. Sollfrank and U. W. Heinz, Phys. Lett. B 459 (1999) 667 arXiv:nucl-th/9906003.

[33] P. F. Kolb, J. Sollfrank and U. W. Heinz, Phys. Rev. C 62 (2000) 054909 arXiv:hep-ph/0006129.

[34] C. Alt et al. [NA49 Collaboration], Phys. Rev. C 68 (2003) 034903 arXiv:nucl-ex/0303001.

[35] Y. Akiba et al. [E802 Collaboration], Nucl. Phys. A 610, 139C (1996).

[36] R. Bramm, Diploma thesis, Institut für Kernphysik, 2002

[37] H. Appelshauser et al. [NA49 Collaboration], Phys. Rev. Lett. 82, 2471 (1999) arXiv:nucl-ex/9810014.

[38] C. Adler et al. [STAR Collaboration], Phys. Rev. Lett. 87, 112303 (2001) arXiv:nucl-ex/0106004.

[39] E. L. Bratkovskaya, W. Cassing and H. Stöcker, Phys. Rev. C 67, 054905 (2003).

[40] M. Bleicher and H. Stocker, Phys. Lett. B 526 (2002) 309 arXiv:hep-ph/0006147. 